\title{
Some conditions for a class of functions to be completely monotonic
}

\section{Senlin Guo*}

${ }^{*}$ Correspondence:
sguo@hotmail.com
Department of Mathematics,
Zhongyuan University of
Technology, Zhengzhou, Henan
450007, China

\begin{abstract}
In this article, we present a necessary condition and a necessary and sufficient condition for a class of functions to be completely monotonic.

MSC: Primary 34A40; 26D10; secondary 26A48
\end{abstract}

Keywords: necessary condition; necessary and sufficient condition; completely monotonic function; gamma function

\section{Introduction and main results}

Recall [1] that a function $f$ is said to be completely monotonic on

$$
\mathbb{R}^{+}:=(0, \infty)
$$

if $f$ has derivatives of all orders on $\mathbb{R}^{+}$and for all $n \in \mathbb{N}_{0}:=\mathbb{N} \cup\{0\}$

$$
(-1)^{n} f^{(n)}(x) \geq 0, \quad x \in \mathbb{R}^{+}
$$

Here and throughout the paper, $\mathbb{N}$ is the set of all positive integers. The set of all completely monotonic functions on $\mathbb{R}^{+}$is denoted by $C M\left(\mathbb{R}^{+}\right)$.

Bernstein [2] proved that a function $f$ on the interval $\mathbb{R}^{+}$is completely monotonic if and only if there exists an increasing function $\alpha(t)$ on $[0, \infty)$ such that

$$
f(x)=\int_{0}^{\infty} e^{-x t} d \alpha(t)
$$

Also recall [3] that a positive function $f$ is said to be logarithmically completely monotonic on $\mathbb{R}^{+}$if $f$ has derivatives of all orders on $\mathbb{R}^{+}$and for all $n \in \mathbb{N}$

$$
(-1)^{n}[\ln f(x)]^{(n)} \geq 0, \quad x \in \mathbb{R}^{+}
$$

The class of all logarithmically completely monotonic functions on $\mathbb{R}^{+}$is denoted by $\operatorname{LCM}\left(\mathbb{R}^{+}\right)$.

It was proved [4] that a logarithmically completely monotonic function is also completely monotonic.

There is a rich literature on completely monotonic, logarithmically completely monotonic functions and their applications. For more recent work, see, for example, [5-28].

(c) 2015 Guo; licensee Springer. This is an Open Access article distributed under the terms of the Creative Commons Attribution License (http://creativecommons.org/licenses/by/4.0), which permits unrestricted use, distribution, and reproduction in any medium, provided the original work is properly credited. 
The Euler gamma function is defined and denoted for $\operatorname{Re} z>0$ by

$$
\Gamma(z):=\int_{0}^{\infty} t^{z-1} e^{-t} d t
$$

The logarithmic derivative of $\Gamma(z)$, denoted by

$$
\psi(z):=\frac{\Gamma^{\prime}(z)}{\Gamma(z)}
$$

is called the psi or digamma function, and the $\psi^{(k)}$ for $k \in \mathbb{N}$ are called the polygamma functions.

In this article, we give two necessary conditions and a necessary and sufficient condition for a class of functions

$$
f_{a, b, c}(x):=(x+a) \ln x-x-\ln \Gamma(x+b)+c, \quad x \in \mathbb{R}^{+},
$$

where $a, c \in \mathbb{R}, b \geq 0$ are parameters, to be completely monotonic. The main results are as follows.

Theorem 1 A necessary condition for the function $f_{a, b, c}(x)$ to be completely monotonic on the interval $(0, \infty)$ is that

$$
\begin{aligned}
& b-a=\frac{1}{2}, \\
& 0<b \leq \frac{1}{2},
\end{aligned}
$$

and

$$
c \geq \ln \sqrt{2 \pi} .
$$

Corollary 1 A necessary condition for the function $f_{a, b, c}(x)$ to be completely monotonic on the interval $(0, \infty)$ is that

$$
-\frac{1}{2}<a \leq 0 .
$$

Theorem 2 For

$$
b \in\left[\frac{1}{2}-\frac{\sqrt{3}}{6}, \frac{1}{2}\right],
$$

a necessary and sufficient condition for the function $f_{a, b, c}(x)$ to be completely monotonic on the interval $(0, \infty)$ is that

$$
b-a=\frac{1}{2}
$$

and

$$
c \geq \ln \sqrt{2 \pi} .
$$




\section{Lemmas}

We need the following lemmas to prove our main results.

Let the $\alpha$ be real parameters, $\beta$ a non-negative parameter. Define

$$
g_{\alpha, \beta}(x):=\frac{x^{x+\beta-\alpha}}{e^{x} \Gamma(x+\beta)}, \quad x \in \mathbb{R}^{+} .
$$

Lemma 1 (see [11]) If

$$
g_{\alpha, \beta} \in L C M\left(\mathbb{R}^{+}\right)
$$

then either

$$
\beta>0 \quad \text { and } \quad \alpha \geq \max \left\{\beta, \frac{1}{2}\right\}
$$

or

$$
\beta=0 \quad \text { and } \quad \alpha \geq 1 \text {. }
$$

Lemma 2 (see [7]) Let

$$
\beta \in\left[\frac{1}{2}-\frac{\sqrt{3}}{6}, \frac{1}{2}\right]
$$

If

$$
\alpha \geq \frac{1}{2}
$$

then

$$
g_{\alpha, \beta} \in \operatorname{LCM}\left(\mathbb{R}^{+}\right) .
$$

\section{Proof of the main results}

Proof of Theorem 1 If

$$
f_{a, b, c} \in C M\left(\mathbb{R}^{+}\right)
$$

then

$$
f_{a, b, c}(x) \geq 0, \quad x \in \mathbb{R}^{+},
$$

and $f_{a, b, c}(x)$ is decreasing on $\mathbb{R}^{+}$.

It is well known that (see [29, p.47])

$$
\ln \Gamma(x+\beta)=\left(x+\beta-\frac{1}{2}\right) \ln x-x+\frac{\ln (2 \pi)}{2}+O\left(\frac{1}{x}\right), \quad \text { as } x \rightarrow \infty .
$$


Hence

$$
f_{a, b, c}(x)=\left(\frac{1}{2}-b+a\right) \ln x-\ln \sqrt{2 \pi}+c+O\left(\frac{1}{x}\right), \quad \text { as } x \rightarrow \infty .
$$

From (8) and (10), we get

$$
\frac{1}{2}-b+a \geq \frac{\ln \sqrt{2 \pi}-c+O(1 / x)}{\ln x}, \quad \text { as } x \rightarrow \infty .
$$

Since

$$
\frac{\ln \sqrt{2 \pi}-c+O(1 / x)}{\ln x} \rightarrow 0, \quad \text { as } x \rightarrow \infty
$$

from (11) we have

$$
b-a \leq \frac{1}{2}
$$

On the other hand, since $f_{a, b, c}(x)$ is decreasing on $\mathbb{R}^{+}$, from (10), we obtain

$$
\left(\frac{1}{2}-b+a\right) \ln x-\ln \sqrt{2 \pi}+c+O\left(\frac{1}{x}\right) \leq f_{a, b, c}(\tau), \quad \text { as } x \rightarrow \infty,
$$

where, in (14), $\tau$ is a fixed number in $\mathbb{R}^{+}$.

Equation (14) is equivalent to

$$
\frac{1}{2}-b+a \leq \frac{\ln \sqrt{2 \pi}+O(1 / x)-c+f_{a, b, c}(\tau)}{\ln x}, \quad \text { as } x \rightarrow \infty .
$$

It is easy to see that

$$
\frac{\ln \sqrt{2 \pi}+O(1 / x)-c+f_{a, b, c}(\tau)}{\ln x} \rightarrow 0, \quad \text { as } x \rightarrow \infty .
$$

Then from (15) we have

$$
b-a \geq \frac{1}{2}
$$

Combining (13) and (17) gives

$$
b-a=\frac{1}{2} .
$$

From (8), (10), and (18), we obtain

$$
c-\ln \sqrt{2 \pi} \geq O\left(\frac{1}{x}\right), \quad \text { as } x \rightarrow \infty .
$$

Since

$$
O\left(\frac{1}{x}\right) \rightarrow 0, \quad \text { as } x \rightarrow \infty
$$


from (19) we have

$$
c \geq \ln \sqrt{2 \pi} .
$$

We note that

$$
f_{a, b, c}(x)=\ln g_{b-a, b}(x)+c .
$$

If

$$
f_{a, b, c} \in C M\left(\mathbb{R}^{+}\right)
$$

we can verify that

$$
g_{b-a, b} \in \operatorname{LCM}\left(\mathbb{R}^{+}\right) .
$$

By Lemma 1, if

$$
b>\frac{1}{2}
$$

then

$$
b-a \geq b>\frac{1}{2}
$$

which contradicts (18); if

$$
b=0 \text {, }
$$

by Lemma 1 , we get

$$
b-a \geq 1
$$

which is another contradiction to (18). So we have proved that

$$
0<b \leq \frac{1}{2}
$$

The proof of Theorem 1 is thus completed.

Proof of Corollary 1 This follows from (2) and (3).

The proof of Corollary 1 is completed.

Proof of Theorem 2 By Theorem 1, the condition is necessary. On the other hand, by Lemma 2, we see that

$$
g_{b-a, b} \in \operatorname{LCM}\left(\mathbb{R}^{+}\right) .
$$


Then from (22), we have, for $n \in \mathbb{N}$,

$$
(-1)^{n} f_{a, b, c}^{(n)}(x) \geq 0, \quad x \in \mathbb{R}^{+}
$$

In particular,

$$
f_{a, b, c}^{\prime}(x) \leq 0, \quad x \in \mathbb{R}^{+} .
$$

Hence $f_{a, b, c}(x)$ is decreasing on $\mathbb{R}^{+}$.

By (9),

$$
f_{a, b, c}(x)=\left(\frac{1}{2}-b+a\right) \ln x+c-\ln \sqrt{2 \pi}+O\left(\frac{1}{x}\right), \quad \text { as } x \rightarrow \infty .
$$

If

$$
b-a=\frac{1}{2}
$$

and

$$
c \geq \ln \sqrt{2 \pi},
$$

from (28), we obtain

$$
\lim _{x \rightarrow \infty} f_{a, b, c}(x)=c-\ln \sqrt{2 \pi} \geq 0 .
$$

Therefore

$$
f_{a, b, c}(x) \geq \lim _{x \rightarrow \infty} f_{a, b, c}(x) \geq 0, \quad x \in \mathbb{R}^{+},
$$

which means that (26) is also valid for $n=0$. Hence we have proved that

$$
f_{a, b, c} \in C M\left(\mathbb{R}^{+}\right) .
$$

The proof of Theorem 2 is hence completed.

\section{Competing interests}

The author declares that he has no competing interests.

\section{Acknowledgements}

The author thanks the editor and the referees for their valuable suggestions to improve the quality of this paper.

Received: 3 November 2014 Accepted: 17 December 2014 Published online: 13 January 2015

\section{References}

1. Bernstein, S: Sur la définition et les propriétés des fonctions analytiques d? une variable réelle. Math. Ann.75, 449-468 (1914)

2. Bernstein, S: Sur les fonctions absolument monotones. Acta Math. 51, 1-66 (1928)

3. Atanassov, RD, Tsoukrovski, UV: Some properties of a class of logarithmically completely monotonic functions. C. R. Acad. Bulgare Sci. 41, 21-23 (1988) 
4. Horn, RA: On infinitely divisible matrices, kernels, and functions. Z. Wahrscheinlichkeitstheor. Verw. Geb. 8, 219-230 (1967)

5. Guo, B-N, Qi, F: A class of completely monotonic functions involving divided differences of the psi and tri-gamma functions and some applications. J. Korean Math. Soc. 48, 655-667 (2011)

6. Guo, S: A class of logarithmically completely monotonic functions and their applications. J. Appl. Math. 2014, 757462 (2014)

7. Guo, S: Logarithmically completely monotonic functions and applications. Appl. Math. Comput. 221, 169-176 (2013)

8. Guo, S: Some properties of completely monotonic sequences and related interpolation. Appl. Math. Comput. 219, 4958-4962 (2013)

9. Guo, S, Laforgia, A, Batir, N, Luo, Q-M: Completely monotonic and related functions: their applications. J. Appl. Math 2014, $768516(2014)$

10. Guo, S, Qi, F: A class of logarithmically completely monotonic functions associated with the gamma function. J. Comput. Appl. Math. 224, 127-132 (2009)

11. Guo, S, Qi, F, Srivastava, HM: A class of logarithmically completely monotonic functions related to the gamma function with applications. Integral Transforms Spec. Funct. 23, 557-566 (2012)

12. Guo, S, Qi, F, Srivastava, HM: Supplements to a class of logarithmically completely monotonic functions associated with the gamma function. Appl. Math. Comput. 197, 768-774 (2008)

13. Guo, S, Qi, F, Srivastava, HM: Necessary and sufficient conditions for two classes of functions to be logarithmically completely monotonic. Integral Transforms Spec. Funct. 18, 819-826 (2007)

14. Guo, S, Srivastava, HM: A certain function class related to the class of logarithmically completely monotonic functions. Math. Comput. Model. 49, 2073-2079 (2009)

15. Guo, S, Srivastava, HM: A class of logarithmically completely monotonic functions. Appl. Math. Lett. 21, 1134-1141 (2008)

16. Guo, S, Srivastava, HM, Batir, N: A certain class of completely monotonic sequences. Adv. Differ. Equ. 2013, 294 (2013)

17. Guo, S, Srivastava, HM, Cheung, WS: Some properties of functions related to certain classes of completely monotonic functions and logarithmically completely monotonic functions. Filomat 28, 821-828 (2014)

18. Krasniqi, VB, Srivastava, HM, Dragomir, SS: Some complete monotonicity properties for the $(p, q)$-gamma function. Appl. Math. Comput. 219, 10538-10547 (2013)

19. Mortici, C: Completely monotone functions and the Wallis ratio. Appl. Math. Lett. 25, 717-722 (2012)

20. Qi, F, Luo, Q-M: Bounds for the ratio of two gamma functions - from Wendel?s and related inequalities to logarithmically completely monotonic functions. Banach J. Math. Anal. 6, 132-158 (2012)

21. Qi, F, Luo, Q-M, Guo, B-N: Complete monotonicity of a function involving the divided difference of digamma functions. Sci. China Math. 56, 2315-2325 (2013)

22. Salem, A: An infinite class of completely monotonic functions involving the $q$-gamma function. J. Math. Anal. Appl. 406, 392-399 (2013)

23. Salem, A: A completely monotonic function involving $q$-gamma and q-digamma functions. J. Approx. Theory 164 , 971-980 (2012)

24. Sevli, H, Batir, N: Complete monotonicity results for some functions involving the gamma and polygamma functions. Math. Comput. Model. 53, 1771-1775 (2011)

25. Shemyakova, E, Khashin, SI, Jeffrey, DJ: A conjecture concerning a completely monotonic function. Comput. Math. Appl. 60, 1360-1363 (2010)

26. Wei, C-F, Guo, B-N: Complete monotonicity of functions connected with the exponential function and derivatives. Abstr. Appl. Anal. 2014, 851213 (2014)

27. Yang, S: Absolutely (completely) monotonic functions and Jordan-type inequalities. Appl. Math. Lett. 25, 571-574 (2012)

28. Srivastava, HM, Guo, S, Qi, F: Some properties of a class of functions related to completely monotonic functions. Comput. Math. Appl. 64, 1649-1654 (2012)

29. Erdélyi, A (ed.): Higher Transcendental Functions, vol. 1. McGraw-Hill, New York (1953)

\section{Submit your manuscript to a SpringerOpen ${ }^{\circ}$ journal and benefit from:}

- Convenient online submission

Rigorous peer review

- Immediate publication on acceptance

- Open access: articles freely available online

- High visibility within the field

- Retaining the copyright to your article 